Allgemeine Beurteilungsgrundsätze im Sinne von $\S$ 94 BetrVG sind Regelungen, die die Bewertung des Verhaltens oden der Leistung eines Arbeitnehmers verobjektivieren und nach einheitlichen Kriterien ausrichten sollen. Es geht damit um die Regelung der Frage, wie der Arbeitnehmer in seiner Leistung oder seinem Verhalten verurteilt werden soll. Mit solchen allgemeinen Beurteilungsgrutidsätzen soll ein einheitliches Vorgehen bei der Beurteilung und ein Bewerten nach einheitlichen Maßstäben ermöglicht und so erreicht werden, daß die Beurteilungsergebnisse miteinander vergleichbar sind (Beschluß des Senats vom 23. Oktober 1984 - 1 ABR 2/83 - zur Veröffentlichung vorgesehen).

Die in den Funktionsbeschreibungen festgelegten Funktionen der Arbeitnehmer können eine tatsächliche Grundlage für die Leistungsbeurteilung abgeben. $\mathrm{Ob}$ sie dazu verwendet werden sollen oder ob eine $\mathrm{Be}$ urteilung auf andere Kriterien aufbauen soll, ist dann zu entscheiden, wenn Beurteilungsgrundsätze vereinbart werden sollen. Ein Mitbestimmungsrecht des Betriebsrats schon bei der Erstellung der Funktionsbeschreibung als möglicher Grundlage einer Leistungsbeurteilung gewährt $\S 94 \mathrm{Abs}$. 2 BetrVG nicht. So hat der Senat auch entschieden, daß das Verlangen des Arbeigebers, Arbeitnehmer sollten ihre Tätigkeiten in Erfassungsbögen eintragen, nicht deswegen ein Mitbestimmungsrecht des Betriebsrats nach $\S 94$ Abs. 2 BetrVG begründet, weil diese Eintragungen tatsächliche Grundlage für eine Leistungsbeurteilung sein können (BAG 37, $112=$ AP Nr. 3 zu $§ 87$ BetrVG 1972 Ordnung des Betriebes).

d) Für Anforderungsprofile hat der Senat entschieden, daß diese keine Auswahlrichtlinien nach $\S 95$ BetrVG sind und deren Erstellung daher nicht dem Mitbestimmungsrecht des Betriebsrats unterliegt (BAG 43, 26 = AP Nr. 2 zu $§ 95$ BetrVG 1972). Die Funktionsbeschreibungen enthalten noch nicht einmal Anforderungen hinsichtlich der fachlichen und persönlichen Voraussetzungen der jeweiligen Funktionsträger.
Ein Mitbestimmungsrecht des Betriebsrats an ihrer Erstellung kann daher schon deswegen aus $\S 95$ BetrVG nicht hergeleitet werden.

2. Die Funktionsbeschreibungen selbst besagen nicht, welcher Tarifgruppe der jeweilige Funktionsträger zuzuordnen ist. Sie enthalten auch keine Angaben darüber, welche Gehaltsbandbreite für den jeweiligen Funktionsträger maßgebend sein soll. Diese Entscheidungen des Arbeitgebers werden vielmehr erst nach Erstellung der Funktionsbeschreibungen getroffen. Sie sind Teil ihrer Ninzung oder Anwendung, die nieht Gegenstand des Rechtsbeschwerdeverfahrens ist. Der Senat kann daher nicht darüber entscheiden, ob die Zuordnung von Gehaltsbandbreiten zu den einzelnen Funktionsbeschreihungen der Mitbestimmung des Betriebsrats unterliegt oder ob die Zuordnung der einzelnen Funktionsträger zu bestimmten Tarifgruppen ein Vorgang ist, der über eine Ein- oder Umgruppierung des Fumktionsträgers hihausgeht und deswegen einem weitergehenden Mitbestimmungsrecht des Betriebsrats unterliegt, als es sieh aus $\S 99$ BetrVG ergibt und vom Arbeitgeber auch zugestanden wird. Nicht zu entscheiden ist darüber, ob der Betriebsrat Regelangen verlangen kann, die ein Beschwerderecht des Arbeitnehmers gegen die Beschreibung seiner Funktion begründen und die den Arbeitnehmern vor Nachteilen einer mögliehen Herabgruppierung schützen.

3. Daß schließlich die bloße Zurverfügungstellung der fertig erstellten Funktionsbeschreibungen im Betrieb als interne Verwaltungsmaßnahme nicht der Mitbestimmung des Betriebsrats unterliegt, bedarf keiner Begründung. Insoweit hat der Betriebsrat auch kein Mitbestimmungsrecht geltend gemacht.

Soweit daher das Landesarbeitsgericht Mitbestimmungsrechte des Betriebsrats bei der Erstellung von Funktionsbeschreibungen und deren Zurverfügungstehung an den Betrieb verneint hat, hat es zutreffend entschieden. Die Rechtsbeschwerde des Betriebsrats gegen diese Entscheidung war daher zurückzuweisen.

\title{
STEUERRECHT
}

\section{Standardsoftware als immaterielles Wirtschaftsgut}

\section{BFH, Urteil vom 3. Juli 1987 (III R 7/86)}

\section{Leitsatz}

Computerprogramme (hier Anwender-Standardsoftware) sind immaterielle Wirtschaftsgüter; für ihre Anschaffung kann daher keine Investitionszulage nach § 19 BerlinFG gewährt werden. (Bestätigung des BFHUrteils vom 5 . Oktober 1979 III R 40/76, BFHE 129, 110, BStBI II 1980, 17),

\author{
Paragraphen \\ BerlinFG $\& 19$.
}

\section{Stichworte}

Anwenderstandardsoftware - Wirtschaftsgut (immaterielles); Computerprogramm - Investitionszulage; Stoftware - Steuerrecht; Standardsoftware - Wirtschaftsgut (immaterielles) 


\section{Zum Sachverhalt}

Ein Berliner Fabrikationsunternehmen (die Klägerin) hatte im Jahre 1979 eine Datenverarbeitungsanlage sowie folgende Anwenderprogramme (Standardprogramme) erworben:

- Auftragsbearbeitung und Materialwirtschaft (Vergütung DM 17.000,-),

- Löhne und Gehälter (Vergütung DM 2.000,-),

- Finanzbuchhaltung (Vergütung DM 4.000,-).

Dem Antrag auf Gewährung einer Invenstitionszulage gemäß $\S 19$ Berlinförderungsgesetz gab das beklagte Finanzamt nur hinsichtlich der Hardware statt. Die gegen die ablehnende Entscheidung hinsichtlich der Software gerichtete Klage blieb erfolglos. Auf die hiergegen eingereichte Revision hob der Bundesfinanzhof die Entscheidung des Finanzgerechts auf und verwies den Streit zur erneuten Verhandlung und Entscheidung an das Finanzgericht zurück; dieses habe den Inhalt der zwischen dem Hersteller des Programms und der Klägerin getroffenen Vereinbarung zu prüfen und zu untersuchen, ob der wirtschaftliche Verkehr die ursprünglich geistige Leistung inzwischen als materialisiert betrachte.

Die daraufhin seitens des Finanzgerichts eingeholten Auskünfte haben im wesentlichen folgendes ergeben:

- Die Industrie- und Handelskammer Berlin hat in einem Gutachten vom 11. 10. 1984 für anwendungsorientierte datenträgergebundene fixe Standardprogramme, die in größerer Auflage auf dem Markt erscheinen, deren Preis im Verhältnis zu den Entwicklungskosten sehr gering ist und die in der Regel über Zwischenhändler „über den Ladentisch“ vertrieben werden, die Qualifizierung als materielles Wirtschaftsgut bejaht. Hinsichtlich des relativ großen Bereichs zwischen Individualsoftware und dieser als Massenprodukt anzusehenden Standardsoftware sei keine allgemein gültige Einordnung möglich.

- Die Programmhersteller haben dem Finanzgericht auf Anfrage folgendes mitgeteilt:

a) Die Entwicklungskosten des Standardprogramms „Auftragsbearbeitung und Materialwirtschaft" hätten mehrere 100000 DM betragen. Ein Datenträger, auf dem dieses Programm dem Anwender zur Verfügung gestellt werde, koste zwischen 10 DM (Floppy-Disk) und 2000 DM (Plattenstapel). Das Programm sei in den Jahren 1977/ 78 entwickelt und ab Mitte 1978 an Anwender abgegeben worden. Dem Anwender werde die Modifizierung eines Programms untersagt, weil nur so der Ersteller dle Gewährleistungsphıcht übernehmen könne. Außerdem erhalte der Anwender in der Regel nur ein Nirtzungsrecht an dem Programm, das es ihm gestatte, das Programm in unveränderter Form auf seiner EDV-Anlage zu nutzen.

b) Das Programm „Löhne und Gehälter" werde seit Januar 1979 geliefert und sei ca. hunderımal installiert worden. Der Preis für das Programm sei ein Lizenzpreis, welcher die zeitlich unbegrenzte Nutzung des Programms beinhalte. Die Installation des
Programms und die Lieferung von Datenträgern würden normalerweise separat berechnet. Die Kosten der Programmentwicklung könnten rechnerisch nicht dem Lizenzpreis gegenübergestellt werden, da der Lizenzpreis vom Markt diktiert werde. c) Das Programm „Finanzbuchhaltung“ sei bisher mehr als hundertmal verkauft worden. Da die Herstellerfirma verpflichtet sei, das Programm den jeweils gültigen Vorschriften der Finanzverwaltung anzupassen, würden dem Anwender eigene Änderungen untersagt.

Auch nach Einhohung dieser Einkünfte hat das Finanzgericht die Klage erneut abgewiesen.

\section{Aus den Gründen:}

Auch die Revision ist unbegründet. Das FG hat zu Recht die von der Klägerin erworbenen Programme nicht als materielle (körperliche), sondern als immaterielle (unkörperliche) Wirtschaftsgüter beurteilt, und es deshalb zu Recht abgelehnt, für den Erwerb dieser Programme Investitionszulage zu gewähren.

\section{Immaterielle Wirtschaftsgüter sind nicht investitionszulagefähig}

1. Der BFH legt zunächst dar, daß für immaterielle Wirtschaftsgüter eine Investitionszulage gemäß $\S 19$ Berlinförderungsgesetz niicht in Betracht karnmt.

\section{Überblick über die bisherige Rechtsprechung des Bundesfinanzhofs zu Individual- und Standardprogrammen}

2. Der Senat hat in seinen Urteilen vom 5. Oktober 1979 III R 78/75 (BFHE 128, 575, BStB1 II 1980, 16), und III R 40/76 (BFHE 129, 110, BStBI II 1980, 17) sowohl Individualprogramme als auch Standardprogramme als immaterielle Wirtschaftsgüter angesehen. In seinem zurückverweisenden Urteil vom 17. Dezember 1982 (vgl. auch Urteil in BFHE 138, 126 BStBl II 1983,647 ) hat der Senat die Auffassung vertreten, daß Gesichtspunkte für die Zuordnung von Standardprogrammen aus der Rechtsnatur der zwlschen dem Ersteller des Programms und dem Anwender abgeschlossenen Verträge (Mietverträge, Lizenzverträge, Kaufverträge) sowie aus den Marktverhältnissen (datenträgergebundene fixe Standardprogramme nach Walter, a.a.O.) gewonnen werden könnten.

a) Mit der Anknüpfung an die Rechtsnatur des im Einzelfall abgeschlossenen Software-Vertrags erwartete der Senat, daß in einet Vielzahl von Fällen eine praktikable und zutreffende steuerliche Einordnung von Standardsoftware vorgenommen werden könne. Denn Standardprogramme wurden in der Praxis in aller Regel aufgrund von Verträgen überlassen, die sich als Nutzungsverträge darstellten (vgl. Kindermann, Gewerblicher Rechtsschutz und Urheberrecht - GRUR - 1983, 150). Reine Kaufverträge bildeten die Ausnahme. Bei Nutzungsverträgen sprach die Vermutung für die Anschaffung eines immateriellen Wirtschaftsguts, weil nach der Rechtsprechung des BFH Nutzungsrechts, gleichgültig, ob Gegenstand der Nut- 
zungsüberlassung materielle oder immaterielle Wirtschaftsgüter sind, bilanzsteuerrechtlich und damit mangels abweichender Begriffsbestimmung auch investitionszulagenrechtlich als immaterielle Wirtschaftsgüter zu qualifizieren sind (z.B. BFH-Urteile vom 1. August 1968 I 206/65, BFHE 94, 52, 55, BStBl II 1969, 66; vom 2. August 1983 VIII R 170/78, BFHE 139, 76, 78, BStBI II 1983, 735); hingegen erschien bei Kaufverträgen die Annahme, der Anwender habe ein materielles Wirtschaftsgut erworben, jedenfalls nicht ausgeschlossen.

b) Mit dem von Walter in die Diskussion eingeführten Begriff der "datenträgergebundenen fixen Standardprogramme" schien sich eine Möglichkeit anzubieten, auf dem Markt bereits als „Massenware" vorhandene Standardsoftware als imaterielle Wirtschaftsgüter zu qualifizieren und diese gegenüber den übrigen Standardprogrammen als immaterielle Wirtschaftsgüter abzugrenzen.

c) Der Senat hält an seinen in den Urteilen vom 3. Dezember 1982 III R 132/81 (BFHE 138, 126, BStB1 II 1983, 647) und vom 17. Dezember 1982 III R $87 / 82$ genannten Kriterien nicht mehr fest.

Die von den Parteien gewählte Vertragsform (z. B. einerseits Lizenzvertrag, andererseits Kaufvertrag) bietet für die steuerliche Beurteilung kein geeignetes Abgrenzungskriterium

3. a) Nach inzwischen gewonnenen Erkenntnissen erweist sich das Vertragsrecht als keine sichere Beurteilungsgrundlagé für die steuerliche Einordnung der Software. Denn das Vertragsrecht ist im Fluß (vgl. Koch, Computer-Vertragsrecht 1986, S 127). Die Gründe liegen im teils ungeklärten, teils unzulänglichen Rechtsschutz für Computerprogramme und in der starken Verbreitung der Personalcomputer in den letzten Jahren. Die in der Praxis bisher üblichen Nutzungsverträge standen ersichtlich unter dem Eindruck, daß Computerprogramme urheberrechtlich geschützt seien (oder doch vertraglich in gleicher Weise geschützt sein sollten). Das war in Schrifttum und Rechtsprechung auchvorherrschende Meinung (vgl. Kolle, GRUR 1982, 443, sowie Koch, a.a.O., S. $205 \mathrm{f}$. mit einer zusammenfassenden Darstellung der Rechtsprechung). Der Bundesgerichtshof (BGH) hat in seinem Urteil vom 9. Mai 1985 I ZR 52/83 (Neue Juristische Wochenschrift - NJW - 1986, 192) jedoch entschieden, daß an Computerprogrammen ein Urheberrecht nur besteht, wenn die Leistung des Programmierers über das Schaffen eines Durchschnittsprogrammierers hinausreicht und damit einen hinreichenden schöpferischen Eigentümlichkeitsgrad i.S. von $\S 2$ Abs. 2 des Urheberrechtsgesetzes (UrhG) erreicht. Seitdem wird die Urheberrechtsschutzfähigkeit von Computerprogrammen in der Literatur sehr zurückhaltend beurteilt (vgl. Bauer, Computer und Recht, 1985, 5). Es bleibt abzuwarten, wie sich diese Entwicklung auf die künftige Vertragsgestaltung auswirkt. Auch die rasche Ausbreitung des Personalcomputers kann die Vertragsgestaltung dahin beeinflussen, daß die Vertragsform für auf Mikrocomputer einsetzbare Standardsoftware künftig im allgemeinen nicht mehr der Nutzungsvertrag, sondern der Kaufvertrag sein wird. Das FG hat sich im Streitfall auch außerstande gesehen, seine Entscheidung auf die abgeschlossenen Verträge zu stützen. Denn nach Auffassung des FG enthielten diese Verträge keine datenverarbeitungsrechtlichen Besonderheiten, die zur Lösung der Streitfrage etwas hätten beitragen können. Dies ist auch der Eindruck des Senats aus weiteren bei ihm abhängigen Verfahren. So gehen die Ersteller von Programmen vielfach von der Einräumung von Nutzungsrechten aus; diese Nutzungsverträge werden von den Zwischenhändlern jedoch nicht "weitergegeben“, so daß sich die Anwender darauf berufen, daß sie die Programme gekauft und zu Eigentum erworben haben. Als entscheidender Gesichtspunkt kommt aber hinzu, daß zivilrechtlich die Einordnung der in der Praxis üblichen Nutzungsverträge nicht gesichert erscheint. Zwar wird dem Anwender nur das nicht ausschließliche und nicht übertragbare Nutzungsrecht an dem Programm übertragen (vgl. $\$ 31$ UrhG für Programme, an denen ein Urheberrecht besteht; für nicht urheberrechtlich geschützte Programme ergeben sich die Einzelheiten des eingeräumten Nutzungsrechtes aus dem jeweiligen Vertrag, vgl. Institut „Finanzen und Steuern“, Die ertragsteuerliche Behandlung von Software, Grüner Brief Nr. 264, S. 41). In der Literatur werden solche Nutzungsverträge als Know-how- oder Lizenzverträge bezeichnet, auf die die Vorschriften über die Pacht anwendbar seien. Die Nutzungsüberlassung erfolgt in diesen Software-Nutzungsverträgen jedoch (im Gegensatz zu Pachtverträgen) unbefristet, d.h. endgültig; auch ist die Einmalvergütung die Regel. Wegen dieser Besonderheiten (unbefristete Nutzungsüberlassung und einmalige Gegenleistung) hat der BGH in seinem Urteil vom 25. März 1987 VIII ZR 43/86 (DB 1987, 1290) die Möglichkeit angedeutet, daß solche Verträge nach Kaufrecht zu beurteilen seien. Auch wird in solchen Fällen angenommen, daß der Anwender an dem überlassenen programm wirtschaftliches Eigentum erwirbt. Geht man hiervon aus und berücksichtigt man zusätzlich, daß Gegenstand eines Kaufvertrags nach allgemeiner Auffassung nicht nur die in $\$ 433$ des Bürgerlichen Gesetzbuches (BGB) ausdrücklich erwähnten Sachen und Rechte, sondern vielmehr verkehrsfähige Güter jeglicher Art wie z.B. ungeschützte Erfindungen, knowhow u.ä., also immaterielle Werte sein können (z.B. Palandt, Bürgerliches Gesetzbuch, 45. Aufl., § 433 Anm. 1 c, cc; Jauernig, Bürgerliches Gesetzbuch, 4. Aufl., $\S 433$ Anm. 5 c; Engel, Betriebs-Berater - BB1985, 1159/1162, m.w.N. in den Fußnoten 33-35), so wird ohne weiteres einsichtig, daß allein aus der zivilrechtlichen Qualifikation eines Software-Vertrages z.B. als Kaufvertrag noch kein zwingender Rückschluß darauf möglich ist, daß der Anwender durch den Abschluß dieses Software-Vertrags kein immaterielles, sondern ein materielles Wirtschaftsgut angeschafft hat und deshalb Investitionszulage erhalten kann. 
b) Auch der von Walter (Der Betrieb - DB $1980,1766,1815)$ in die Diskussion eingeführte Begriff des „datenträgergebundenen fixen Standardprogramms“ ist für eine Abgrenzung nicht geeignet ... (wird ausgeführt).

c) Ungeeignet sind auch die von der Industrieund Handelskammer Berlin in ihrem Gutachten vom 11. Oktober 1984 genannten Abgrenzungskriterien: Auflagenhöhe, Verhältnis von Entwicklungskosten zu Einzelerwerbspreis sowie der Vertriebsweg. So sehr diese Merkmale im einzelnen und in ihrer Gesamtheit bestechend erscheinen, weil sich einen Markt kennzeichnen, auf dem - wenigstens in Teilbereichen - bereits ein Gut den Charakter einer „Ware“ u.U. sogar einer „Massenware“ angenommen hat, so sind diese Merkmale für eine sichere Abgrenzung in der Praxis doch nicht brauchbar (ebenso Institut „Finanzen und Steuern“, a.a.O., S. 68 f.). Die Gründe dafür sind nach Auffassung des Senats so evident, daß sie im einzelnen hier nicht dargestellt zu werden brauchen. Diese Merkmale erwiesen sich auch im Streitfall als so wenig ergiebig, daß das FG seine Entscheidung darauf nicht stützen konnte.

Software ist immaterielles Wirtschaftsgut, da der geistige Gehalt des Programms im Vordergrund steht

4. Das FG hat den Erwerb der streitigen Programme als Anschaffung immaterieller Wirtschaftsgüter gewertet, weil es die Programme als geistig-schöpferische Werke ansah und davon ausging, daß der Anwender das Entgelt für dieses geistige Werk bezahlt. Der Senat tritt dieser Auffassung bei. Er sieht dabei im Ergebnis zwischen Individualprogrammen und Standardprogrammen keinen Unterschied.

\section{Überragende wirtschaftliche Bedeutung des Programminhalts}

a) Bei der Wertung eines Computerprogramms steht sein geistiger Gehalt im Vordergrund. An ihm ist der Anwender in erster Linie interessiert; dafür ist er bereit, u.U. einen hohen Preis zu zahlen. Das Programm stellt für ihn einen betrieblichen Vorteil dar, indem es ihn befähigt, in seinem Betrieb oder Büro vielfältige Aufgaben zu erledigen: z. B. mathematische, wissenschaftliche und technische Berechnungen durchzuführen, Produktionsvorgänge zu steuern, Kalkulationen und Buchführungsaufgaben zu lösen, Texte zu schreiben u. ä. (vgl. Engel, BB 1985, 1159). Computerprogramme sind anderen wirtschaftlichen Gütern ähnlich, wie technischen Erfindungen und geistigen Schöpfungen im Sinne des Urheberrechts, die unstreitig immaterielle Wirtschaftsgüter sind. Von Bedeutung ist in diesem Zusammenhang auch, daß Datenverarbeitungsprogramme nach $\S 2$ Abs. 1 UrhG urheberrechtlich geschützte Werke sein können.

Dem Programminhalt kommt die überragende wirtschaftliche Bedeutung zu. Der Datenträger tritt demgegenüber zurück. Er dient nur dazu, das
Programm unverlierbar zu machen und es als Gut im wirtschaftlichen Verkehr umsetzen zu können, sowie als Eigengabemedium für den Computer. Sein Materialwert steht im Regelfall auch in keinem Verhältnis zum Programm und muß daher grundsätzlich außer Betracht bleiben.

\section{Befristete Nutzungsrechte}

b) Soweit Computer-Programme und die sie speichernden Datenträger (Vervielfältigungsstücke) aufgrund von Verträgen "geliefert" werden, die nicht nur, wie in der Praxis bisher weithin üblich, als Nutzungsoder Lizenzverträge bezeichnet sind, sondern auch tatsächlich dem Anwender nur ein befristetes Nutzungsrecht an dem Programm als geistiger Leistung und dem dazugehörigen Datenträger als Sache einräumen, ist im Hinblick auf die oben zu 2. a) erwähnte Qualifizierung von Nutzungsrechten als immaterielle Wirtschaftsgüter ohne weiteres einsichtig, daß der Anwender, im Streitfall die Klägerin, kein materialles, sondern ein immaterielles Wirtschaftsgut, eben ein Nutzungsrecht an einer geistigen Leistung und der sie speichernden Sache angeschafft und daher mit dem Nutzungsentgelt nur Anschaffungskosten für ein immaterielles Wirtschaftsgut aufgewendet hat, dessen Anschaffung nach $\S 19$ BerlinFG nicht begünstigt ist.

\section{Kauf unbefristeter Rechte}

c) Der Senat kann jedoch zugunsten der Klägerin ohne weiteres unterstellen, daß diese die drei Computer-Programme zusammen mit den sie speichernden Datenträgern (d.h. den Vervielfältigungsstücken der Originalkopie) „käuflich“ erworben und für diesen Erwerb ein Entgelt bezahlt hat (vgl. zur evtl. Anwendung von Kaufrecht auf einen Software-Vertrag bei unbefristeter Nutzungsüberlassung BGH-Urteil vom 25. März 1987 VIII ZU 43/86, BB 1987, 1277, 1279 Spalte 1 oben). Gleichwohl hat die Klägerin damit keine materiellen, sondern immaterielle Wirtschaftsgüter angeschafft; ihre Aufwendungen sind keine Anschaffungskosten für „bewegliche Wirtschaftsgüter“ (Sachen), sondern Anschaffungskosten für immaterielle Wirtschaftsgüter, nämlich die Programme. Denn auch soweit Software-Verträge zivilrechtlich als Kauifverträge (oder kaufrechtsähnliche Austauschverträge) zu qualifizieren sind, ist Gegenstand des Kaufvertrags (Austauschvertrags) keine Sache (i.S. von $\S 90$ BGB), sondern - mindestens weitaus primär - ein immaterielles Gut, nämlich das Programm als Werk mit geistigem Inhalt. Der Käufer will mit Abschluß des Software-Vertrags die rechtliche und wirtschaftliche Macht erlangen, das Programmals Werk mit geistigem Inhalt für seine betrieblichen Zwecke einsetzen zu können; das sachenrechtliche Eigentum am Datenträger ist für ihn bei weitem sekundär und nicht das eigentliche Kaufziel. Das immaterielle Gut „Programm“, und nicht etwa die Sache „Datenträger" ist es, wofür er den u.U. hohen Kaufpreis vereinbart und bezahlt (vgl. z. B. Engel, BB 1985, 
$1159 / 1160$ und 1162, m.w. N. in den Fußnoten 10 und 32; Mehrings, NJW 1986, 1904/1905; ähnlich BrandiDohrn, Computer und Recht - CuR - 1986, 63, 66; vgl. auch BGH-Urteil vom 25. März 1987 VIII ZU 43/ 86, BB 1987, 1277, 1278 Spalte 2 Absatz 4). Demgemäß sind bei Mängeln (Fehlern) des Programms die Vorschriften der $\$ \$ 459 \mathrm{ff}$. BGB über die Sachmängelhaftung jedenfalls nicht un mittelbar anwendbar, weil diese tatbestandlich den Kauf einer Sache und einen Mangel "der verkauften Sache“ voraussetzen (vgl. Engel, a.a.O., S. 1164; Mehrings, a.a.O. S. 1907).

Wenn aber zivilrechtlich Kaufgegenstand, also das Gut, wofür der Kaufpreis vereinbart und bezahlt wird, das Programm als Werk mit geistigem Inhalt und damit ein immaterieller Wert ist, so ist auch investitionszulagenrechtlich davon auszugehen, daß Gegenstand der Anschaffung ein immaterielles Wirtschaftsgut ist und die Aufwendungen des Käufers, im Streitfall der Klägerin, Anschaffungskosten für ein immaterielles Wirtschaftsgut sind:

Dieser Wertung steht nicht entgegen, daß Anwender-Standardprogramme, die käuflich erworben werden, möglicherweise in bestimmten Wirtschaftskreisen als "Waren" bezeichnet und angesehen werden. Der Begriff der Ware ist sowohl im allgemeinen als auch itn gesetzlichen Sprachgebrauch vieldeutig und keineswegs stets auf körperliehe Gegenstände (Sachen) beschränkt (vgl. z.B. Baumbach/Hefermehl, Wettbewerbsrecht, 14. Aufl., $\S 2$ UWG Rz. 1). Aus der Bezeichnung eines Wirtschaftsguts als „Ware“ lassen sich daher keine zwingenden Schlüsse auf seine investitionszulagenrechtliche Einordnung als materielles oder immaterielles Wirtschaftsgut ziehen.

Keine analoge Anwendung der Vorschriften über Investitionszulage auf immaterielle Wirtschaftsgüter

Der BFH prüft im Hinblick darauf, daß zivilrechtlich auf kaufrechtsähmliche Softwareverträge eine analoge Anwendung der Vorschriften über die Sachmängelhaftung befürwortet wird, ob auch eine analoge Anwendung der für materielle Wirtschaftsgüter geltenden Investitionszulagevorschriften auf Computer-Programme in Betracht kommt, lehnt diese Analogie jedoch ab.

\section{Andere Beurteilung evtl. für Trivialprogramme}

e) Über Trivialprogramme hat der Senat nicht zu entscheiden. Für sie gelten möglicherweise andere Grundsätze, weil sich bei ihnen das Wertverhältnis von Programminhali und Programmträger wesentlich verschoben hat. Einen Vergleich dieser Programme mit Büchern oder Schallplatten will der Senat nicht ausschließen. Allerdings könnte die Invesititonszulagefähigkeit für diese Programme an $\S 6 \mathrm{Ab} .2 \mathrm{EStG}$ scheitern.

\section{Programme als unselbständiger Bestandteil eines Gerätes}

Unberührt bleiben von der Senatentscheidung auch technische und elektronische Geräte, in de- ren Gehäuse die Programmsteuerung als unselbständiger Bestandteil eingebaut ist. Hier ist nur das technische Gerät zu beurteilen; es ist ein körperliches (materielles) Wirtschaftsgut und damit zulagebegünstigt.

\section{Anmerkung}

Mit seiner Entscheidung vom 3. 7.1987 setzt der III. Senat des Bundesgerichtshof vorerst einen Schlußstrich unter die zunehmend im Fachschrifttum (vgl. Feldhahn, DStR 85, 336) und vereinzelt in der Rechtsprechung (vgl. FG Berlin, EFG 1986, 106) vertretene Auffassung, daß Standardsoftware bei kaufähnlicher Vertragsgestaltung als materielles Wirtschaftsgut einzuordnen sei. Die Finanzverwaltung hat sich mit dieser Auffassung ohnehim nicht anfreunden können und Standardprogramme nach wie vor als immaterielle Wirtschaftsgüter betrachtet (vgl. Abschnitt 31 a Abs. 1 Satz 3 EStR sowie BMF-Schreiben vom 10. 10. 1983, BStBl 1983, I., 446).

Für die steuerliche Praxis ergeben sich aus dieser Entscheidung im wesentlichen folgende Auswirkungen:

1. Abgrenzung: Individualsoftware und Standardsoftware stellen gleichermaßen immaterielle Wirtschaftsgüter dar. $\mathrm{Ob}$ der Anwender diese Programme auf der Grundlage eines Kauf- oder eines Nutzungsvertrages erhält, ist unerheblich. Lediglich für Trivalprogramme läßt der BFH eine anderweitige Beurteilung offen.

2. Investitionszulage: Für EDV-Programme wird weder nach $\S 19$ Berlinförderungsgesetz noch nach den Vorschriften des Investitionszulagengesetzes eine Investitionszulage gewährt. Einzige Ausnahme ist die Investitionszulage für Forschung und Entwicklung gemäß $§ 4$ Abs. 2 Nr. 3 Investitionszulagengesetz, wonach EDV-Programme zulagefähig sein können, wenn sie „neu“ sind und unmittelbar und ausschließlich der Forschung und Entwicklung dienen.

3. Abschreibungen: Sonderabschreibungen gemäß $\S 82 \mathrm{~d}$ EStDV (Forschung und Entwicklung), $\$ 6$ Abs. 2 EStG (Sofortabschreibung geringwertiger Wirtschaftsgüter), $\S 7 \mathrm{~d}$ EStG (Umweltschutz), $\$ 7 \mathrm{~g}$ EStG (Mittelstandsförderung) und $\S 14$ Berlinförderungsgesetz kommen für Software nicht in Betracht.

4. Aktivierungsfähigkeit: Vom Anwender selbst geschaffene (also nicht von Dritten verkaufte) Software, die nicht zum Verkauf bestimmt, sondern dem Anlagevermögen zuzuordnen ist, darf in der Bilanz nicht als Aktivposten angesetzt werden. Die Herstellungskosten für die Software führen mithin sofort zu Aufwand.

Walter Grosse, Rechtsanwalt und Stewerberater, München 